\title{
Endoscopic Training Model for Intranasal Transsphenoidal Hypophysis Surgery Using a Skull Model and Chicken Wings
}

\author{
Adnan ALTUN ${ }^{1}$, Cengiz COKLUK² \\ ${ }^{1}$ Karatay University, School of Medicine, Department of Neurosurgery, Konya, Turkey \\ ${ }^{2}$ Ondokuzmayis University, School of Medicine, Department of Neurosurgery, Samsun, Turkey \\ Corresponding author: Adnan ALTUN draaltun@hotmail.com
}

\section{ABSTRACT}

AIM: To evaluate the feasibility of an experimentally designed model for training on endoscopic intranasal transsphenoidal hypophysis surgery.

MATERIAL and METHODS: In this experimental study, a surgical training model for endoscopic transsphenoidal hypophysis surgery was designed to simulate real life surgical challenges, such as drilling through the sella turcica under endoscopic vision. This laboratory experiment was repeated at 1 -week intervals and it was observed that surgeons improved their skills. The compatibility of the training model was evaluated as either poor, acceptable or perfect.

RESULTS: The results revealed that according to earlier data, greater success occurred in tests conducted after 1 week. Three new specialist neurosurgeons also expressed that their self-confidence increased during the second procedure.

CONCLUSION: This laboratory study will result in improved use of microsurgical instruments and understanding of the threedimensional surgical field, as well as the development of manual dexterity. We believe that this model will contribute to the practical training of endoscopic hypophysis surgery.

KEYWORDS: Microneurosurgery, Training of endoscopic surgery, Microdrilling, Chicken wings, Hypophysis surgery

\section{INTRODUCTION}

I ntranasal transsphenoidal hypophysis surgery requires enduring a learning curve. The trainee neurosurgeon must get accustomed to endoscopic vision and master the handling of microsurgical equipment under this vision as well as gain the ability and confidence to navigate through this highly specific and critical anatomic region before tackling a real case. This mandates acquisition of a skillset including, but not limited to, adjustment and handling of the neuroendoscope and/or operating microscope, drilling of the cranial bones without damaging the surrounding vital structures, passing neurobiological membranes and performing neurovascular dissection in a very confined space $(1,4,8,10)$. In endoscopic intranasal transsphenoidal hypophysis surgery, bony substance from the cranial base must be sufficiently removed to provide access (7). This is done using a high-speed microdrill while preserving the critical neurovascular structures in the vicinity (3). The floor of the sella turcica must be removed without damaging the internal carotid arteries on either side, unilateral of the dura mater, circular venous sinus and medial wall of the cavernous sinus $(3,7,9)$.

In this experimental study, an endoscopic training model for intranasal transsphenoidal hypophysis surgery was developed by placing one commercially available proximal segment of a chicken wing (which contained the humerus) inside a specially tailored plastic replica of a human skull. The construct mimics the biological barriers that the surgeon needs to overcome to access and ablate a hypophyseal mass. Structurally, the bone of the chicken wing represents the bony structure of the sellar floor. The fine skin overlying the chicken bone represents the dura mater overlying the sella turcica. The brachial vessels coursing along the bone represent the 
critical vascular structures to be preserved. The cortex of the chicken humerus represents the base of the sella and its medulla is the proposed tumour to be ablated $(5,6)$. In this report, experimental findings, pitfalls, practical feasibility, and suggestions were discussed with respect to the literature.

\section{MATERIAL and METHODS}

Surgeries were performed under endoscopic vision. The training goals were as follows: successfully handling the endoscope in a manner to acquire a clear image via the nasal speculum, accessing the bone by dissection of the skin while preserving the vascular structure, removal of the bone cortex with a high-speed microsurgical drill and removal of the bone marrow via a pituitary curette without damaging the vessels in the vicinity.

\section{Setup}

A commercially available skull model (Sawbones Inc., WA, USA) was specially tailored to accommodate the training model. The sphenoidal sinus and the sella turcica were cut out using a household power drill. Subsequently, one humeral segment of a chicken wing, commercially available in stores for culinary purposes, was fitted in the space created and further fixed using bone wax. After ensuring that the chicken wing was snugly fitted into place, and was not dislodged by tilting the skull, the model was fixed with the three-pin Mayfield skull fixator system in the hyperextended position. This was similar to the way it is done in a real hypophyseal surgery. The nasal speculum was fitted inside the model's nasal aperture. The endoscope was then introduced. Once a clear endoscopic vision was obtained, surgical training intervention was commenced. The skin of the chicken was superficially incised using a size 11 blade. The brachial vessels overlying the bone were then located, and via microdissection, they were freed and retracted using micro-forceps, a micro-dissector and a microhook. Once the vessels were safely moved, the exposed bony cortex was removed using a high-speed microdrill (MidasRex Legend, Medtronic Inc., MI, USA). Initially, the drill was fitted with a $0.5-\mathrm{mm}$ ball burr. Once the marrow was visible, the bit was changed to a $0.5-\mathrm{mm}$ diamond burr for a more controlled drilling, until a window with a diameter of $7 \mathrm{~mm}$ was created. The hypophyseal curette was then carefully introduced without damaging the vessels and the medullar content of the bone was removed as if it were a pituitary tumour. Subsequently, the training session was terminated.

Three neurosurgeons conducted the experiment. The senior neurosurgeon was very experienced and specialized in pituitary tumour surgery. He had a reputation for tackling difficult and high-risk tumours that are daunting to many others. There were also three new specialist neurosurgeons who did not undergo endoscopic pituitary surgery training. Experiments were conducted using equipment by Storz (Karl Storz, Germany) and microsurgical instruments.

The senior surgeon detailed all experimental models and their uses. Three new surgeons took part in this experimental study.
Thirty chicken wings were used as training models in this experimental feasibility study. Fifteen chicken wings were used in the first week and another fifteen were used as a control group 1 week later. Each experiment was repeated five times, and the average of five tests (poor, acceptable and perfect) was taken for each surgeon.

Evaluation criteria were made under the following four headings: 1) receiving images by endoscope within nasal speculum; 2) while handling the endoscope, to reach the bone by doing dissection and separation without damaging the vascular structure using the other hand; 3) while handling the endoscope, to pass the bone cortex with a high-speed microsurgical drill by using the other hand without damaging the vascular structure; and 4) while handling the endoscope, to remove the bone marrow material with a pituitary curette without damaging the vascular structure by using the other hand. These evaluation criteria were recorded based on the observations of the senior surgeon.

The wings were placed in the hypophysis located inside the skull model before processing. A three-pin Mayfield skull fixator system was then used in the fixation of the head (Figure 1). A nasal speculum was fixed inside the skull model's nasal opening before the surgical intervention began. All steps of this study were performed under endoscopic vision. The skin surfaces of the chicken wings were cut using size 11 surgical blades. Dissection and separation were performed in the opening of the soft tissues of the wings.

Using a high-speed microsurgical drill (Midas Rex ${ }^{\circledR}$ Legend $^{\circledR}$ Electric System, USA), the humerus was drilled with 5-mm ball diamond burrs (Figure 2A, B). Interior membranous dissection and separation were conducted via microforceps, a microdissector and a micro-hook (Figure 3A, B).

One week later, the same surgical team repeated the same experiment under the supervision of a senior surgeon. The results revealed that according to earlier data, greater success occurred in tests conducted after 1 week (Figure 4).

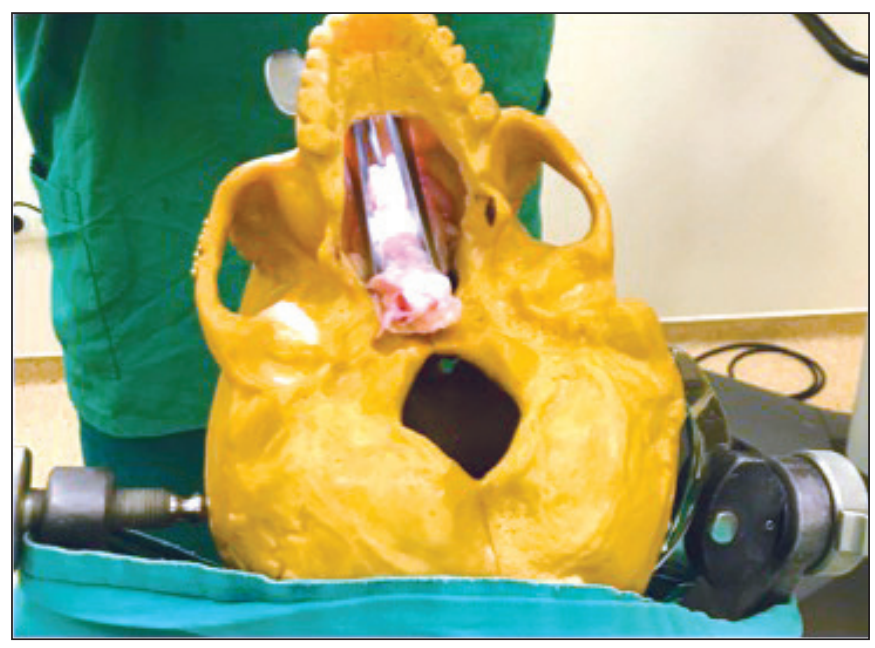

Figure 1: The wings were placed in the hypophysis located inside the skull model before processing. 
The main challenge of the training session was the preservation of vascular structures in the vicinity (criteria 3). The compatibility of the procedure was also evaluated within the three groups of poor, acceptable or perfect.

\section{RESULTS}

Each experiment was repeated five times, and the average of the five tests (poor, acceptable and perfect) was taken for each surgeon. The evaluation criteria were recorded based on the observations of the senior surgeon.

In the first week of the procedure, it was observed that surgeons were most often challenged by the third criterion, and were less challenged by the second, fourth and first criteria in this order. A week later, the same surgical team repeated the same experiment under the supervision of the senior surgeon.
In the second week of the procedure, surgeons were able to further develop their abilities in criteria three and one. Three new specialist neurosurgeons also expressed that their selfconfidence increased during the second procedure.

\section{DISCUSSION}

Training in endoscopic microneurosurgical techniques before actual surgical intervention is crucial for neurosurgeons (4). High resolution, real-time, operative field images can be obtained by using new generation optical lenses and cameras during microneurosurgical interventions in the craniospinal cavities. Orientation of the surgeon to the axial, coronal, and sagittal directions along the surgical intervention requires repetitive practice on experimental models resembling clinical counterparts. Endoscopic surgery is different from microscopic
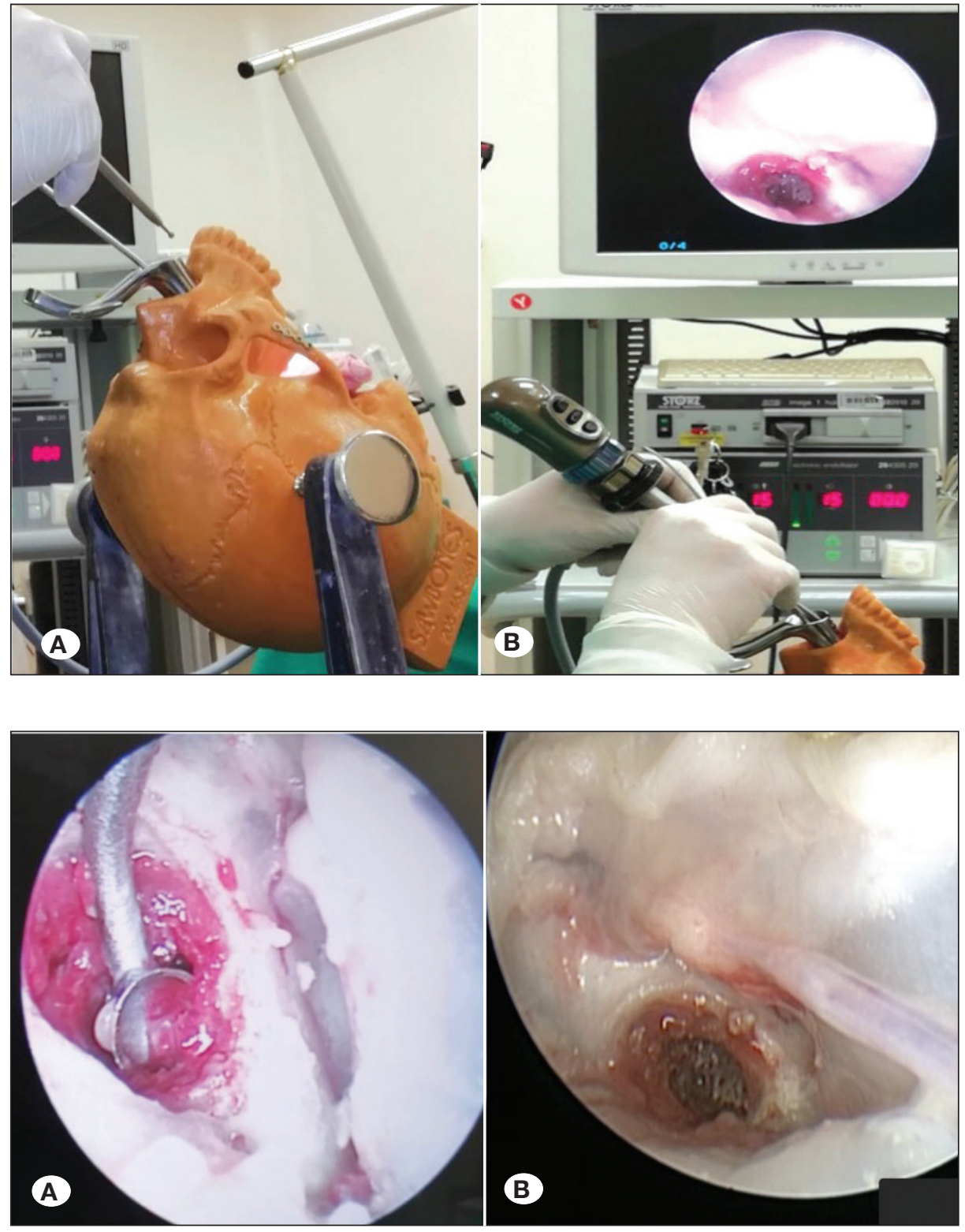

Figure 2: A) Using high-speed microsurgical instruments for opening burr holes. B) All steps of this study were performed under endoscopic vision.
Figure 3: A) Hypophysis curettes were used in the removal of soft materials as a model for hypophysis tumours. B) Soft materials were removed as a model for hypophysis tumours without damaging the vessels. 


\section{first week evaluations}

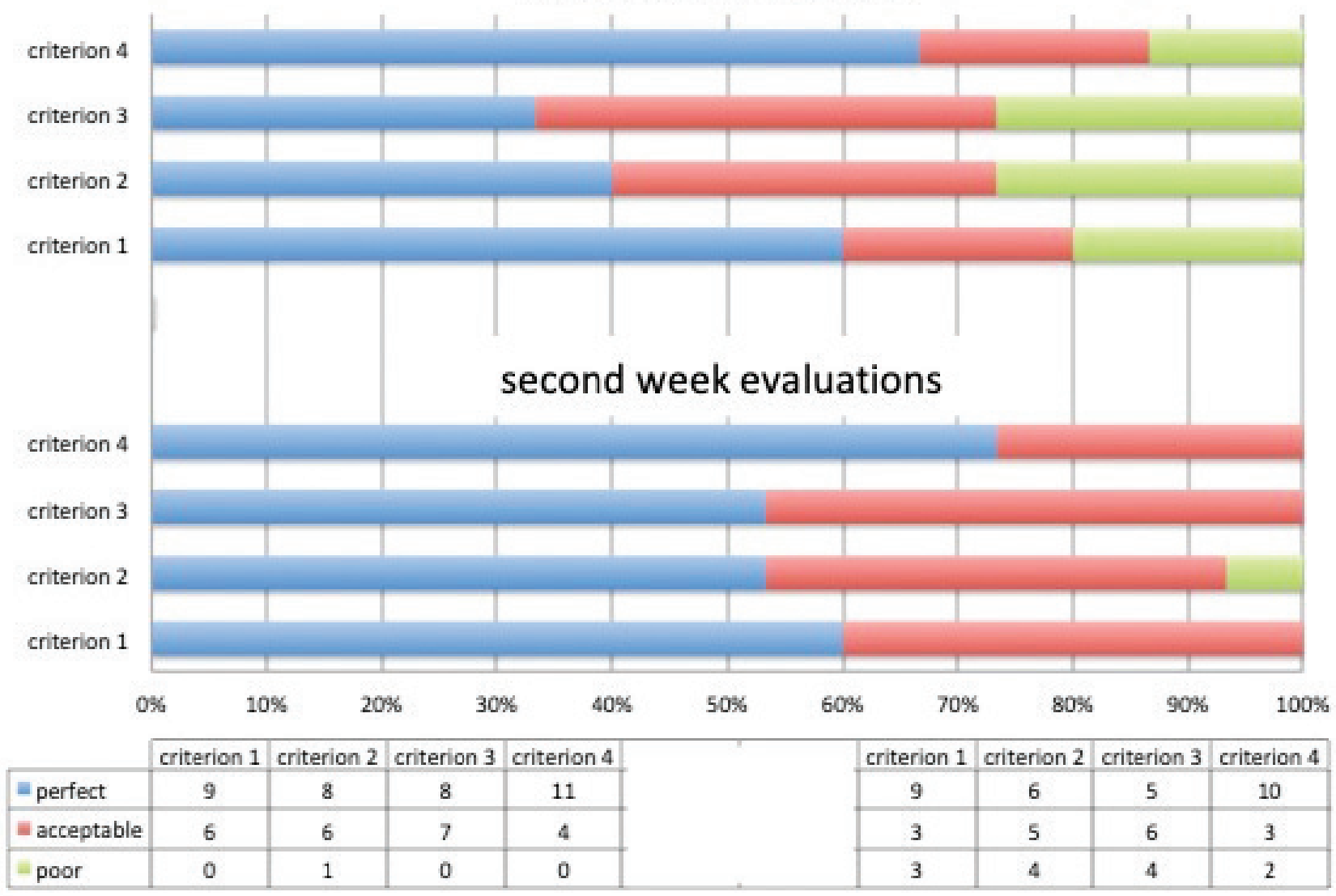

Figure 4: The results of the experimental studies performed at weeks 1 and 2 are based on observation.

intervention in some aspects. A surgical microscope provides a stereoscopic view obtained from the operative field, whereas an endoscope does not. A learning curve is necessary to gain an endoscopic view. To perform safe neurosurgical interventions, regional surgical neuroanatomy and microneurosurgical instruments should be repetitively handled by the surgeon prior to surgery. It is imperative that surgical techniques be mastered in appropriate training models before the trainee tackles a live human patient $(3,4)$. The operating room should not be where a trainee gets accustomed to and is familiarized with high-end surgical equipment such as the operating microscope or endoscope (1-4,8,10). Vascular end-to-end, end-to-side and side-to-side anastomoses as well as sylvian fissure dissection in rat models are frequently performed in the experimental laboratory to improve microneurosurgical ability. For this reason, a training model for endoscopic surgery has been proposed in this study.

An appropriate and successful model should have some similarities with the actual clinical counterpart. The resemblance of the present model to the hypophysis region is limited. This model has internal cavities, an outer membranous structure and bone inside. However, our surgical technique in the microdrilling of the wing's bone resembles microdrilling of the parasellar and sellar bones. Other important considerations include the easily obtainable and cheap properties of the materials. The model must be conveniently prepared without tedious measures.

Live models create some problematic limitations in experimental practice, and ethical issues must be considered. A cadaveric chicken wing is not a living model; therefore, permission for its use in experimental practices is not required from the Ethical Committee (3).

Chicken wing is a readily available and inexpensive foodstuff. These features boast its practical advantages as a training material (3).

The wing's bone is real bone material. While it is not exactly of the same consistency as that of the skull base, it reacts very similarly to being drilled and fenestrated. It is also amenable to repeated use (3).

The skull model is used together with the chicken wing during the experiment. The wing should be fixed in the cranial base before starting the experiment. Using the Mayfield threepin fixation device is also another important issue for the experiment. The sagittal flexion and extension of the head that is achieved by using the three-pin head fixator is important in gaining the orientation of the hypophysis. 
The high-speed microdrill can be regarded as the most critical of the instruments to be handled. An experimental model, which needs high expertise, is instrumental in the model's efficiency (3). Adjusting the turning rate of the drill is critical to safely performing the procedure. This is a feature that this model delivers (3). Also, the removal of the outermost layer from the membrane located under the wing's surface is another important step. Use of the micro-hook in dissecting the inner membranes must also be executed to complete this training model.

\section{CONCLUSION}

Removal of the thin and delicate bony structure of the cranial base by microdrilling is one of the cardinal steps in endoscopic microneurosurgical interventions (3). Before taking on live cases, this process should be practised several times on surgical training models. This report presents the convenient and inexpensive use of chicken wings as a practical and viable model. Precise usage of the microsurgical instruments along with good surgical practice is essential. We believe that this model will contribute to the practical training of endoscopic microneurosurgery.

\section{REFERENCES}

1. Altunrende ME, Hamamcioglu MK, Hicdonmez T, Akcakaya MO, Birgili B, Cobanoglu S: Microsurgical training model for residents to approach to the orbit and the optic nerve in fresh cadaveric sheep cranium. J Neurosci Rural Pract 5:151-154, 2014
2. Belykh E, Byvaltsev V: Off-the-job microsurgical training on dry models: Siberian experience. World Neurosurg 82:20-24, 2014

3. Cokluk C: Using of chicken eggshell in the microneusurgical training model for microdrilling of sella floor. Clin Surg 2:1512, 2017

4. Cokluk C, Aydin K: Maintaining microneurosurgical ability via staying active in microneurosurgery. Minim Invasive Neurosurg 50:324-327, 2007

5. Hincke MT, Nys Y, Gautron J, Mann K, Rodriguez-Navarro $A B$, McKee MD: The eggshell: Structure, composition and mineralization. Front Biosci (Landmark Ed) 17:1266-1280, 2012

6. Nakano T, Ikawa NI, Ozimek L: Chemical composition of chicken eggshell and shell membranes. Poult Sci 82:510-514, 2003

7. Sai Kiran NA, Furtado SV, Hegde AS: How I do it: Anterior clinoidectomy and optic canal unroofing for microneurosurgical management of ophthalmic segment aneurysms. Acta Neurochir (Wien) 155:1025-1029, 2013

8. Spetzger U, von Schilling A, Brombach T, Winkler G: Training models for vascular microneurosurgery. Acta Neurochir Suppl 112:115-119, 2011

9. Turan Suslu H, Ceylan D, Tatarli N, Hicdonmez T, Seker A, Bayri $\mathrm{Y}$, Kilic T: Laboratory training in the retrosigmoid approach using cadaveric silicone injected cow brain. $\mathrm{Br} \mathrm{J}$ Neurosurg 27:812-814, 2013

10. Yadav YR, Parihar V, Ratre $S$, Kher $Y$, lqbal $M$ : Microneurosurgical skills training. J Neurol Surg A Cent Eur Neurosurg 77:146-154, 2016 